\title{
Association of thyroid function with obesity measured as body fat percentage and insulin resistance
}

\begin{abstract}
Introduction: There is scarce information about the association of thyroid function (TF) with body fat percentage $(\mathrm{BF} \%)$.

Objective: We aimed to evaluate the association between $\mathrm{TF}$ and $\mathrm{BF} \%$. We also evaluated the association of TF with other variables including insulin resistance (IR).

Methods: We included patients from our outpatient clinic with normal TF. A complete evaluation was done, including TF tests: thyroid stimulating hormone (TSH), total triiodothyronine (T3), total thyroxine (T4), $3 \mathrm{hr}$ oral glucose tolerance test (OGTT), and BF\% measured with electrical bioimpedance. We divided the population in 2 groups in terms of $\mathrm{BF} \%$ and compared the $\mathrm{TF}$ in each group. Correlation and linear regression analyses were done to evaluate the association of $\mathrm{TSH}$ and $\mathrm{T} 3$ with $\mathrm{BF} \%$, and IR.

Results: We evaluated 203 patients (35.9\% male). The mean age of the studied subjects was $38.9 \pm 13.5$ years, with a body mass index (BMI) of $32.7 \pm 8.7 \mathrm{~kg} / \mathrm{m}^{2}$, and a mean $\mathrm{BF} \%$ of $38.4 \pm 9.5 \%$. Between men and women, there were no differences in T3, T4, free thyroxine index (FTI), and TSH. In men, obese patients had more T3 $(1.81 \pm 0.53$ vs $1.57 \pm 0.25 \mathrm{nmol} / \mathrm{L}$, $\mathrm{p}=0.04$ ). In women, there were no differences in $\mathrm{T} 3$ concentrations, and obese patients had more TSH $(2.12 \pm 0.9$ vs $1.65 \pm 0.8 \mathrm{mIU} / \mathrm{mL}, \mathrm{p}=0.04)$. After weight adjustment, we identified a significant correlation between $\mathrm{BF} \%$ and TSH $(\mathrm{r}=0.17, \mathrm{p}=0.02)$, and after age and weight adjustment, we identified a significant correlation between T3 and the Homeostasis Model Assessment of insulin resistance (HOMA-IR) $(\mathrm{r}=0.38, \mathrm{p}<0.001)$. In the multiple regression analysis $\mathrm{BF} \%$ and male gender, were independently associated with TSH concentration, and HOMA-IR was independently associated with T3 concentration.
\end{abstract}

Conclusion: TSH is associated with $\mathrm{BF} \%$ independently of weight, and $\mathrm{T} 3$ is associated with HOMA-IR, independently of age and weight.

Keywords: Thyroid function; Body fat percentage; Insulin resistance; Thyroid hormone; Rheumatoid arthritis; Seizures; Fasting insulin; Free thyroxine index
Volume 3 Issue 6 - 2016

\section{Miguel Angel Gomez-Sámano, Clara Elena Meza-Arana, Alfonso Gulias-Herrero \\ Department of Internal Medicine, Salvador Zubiran National Institute of Health Sciences and Nutrition, Mexico}

\begin{abstract}
Correspondence: Alfonso Gulias-Herrero, Department of Internal Medicine, Instituto Nacional de Ciencias Medicas y Nutricion Salvador Zubiran, Vasco de Quiroga \# 15, Belisario Domínguez Sección XVI,Tlalpan I4080, Mexico City, Mexico,Tel 52555556559068, Email alfonsotiranicida@gmail.com
\end{abstract}

Received: October 01, 2016 | Published: December 08, 2016
Abbrevations: TF: Thyroid Function; IR: Insulin Resistance; TSH: Thyroid Stimulating Hormone; T3: Total Triiodothyronine; T4: Total Thyroxine; OGTT: Oral Glucose Tolerance Test; BMI: Body Mass Index; FTI: Free Thyroxine Index; HOMA-IR: Homeostasis Model Assessment of Insulin Resistance; TRH: Thyrotropin Releasing Hormone; BF\%: Body Fat Percentage; INCMNSZ: Instituto Nacional de Ciencias Medicas y Nutricion Salvador Zubiran; TU: Thyroid Uptake; THBR=TU/0.4: Thyroid Hormone Binding Ratio; TG: Thyroglobulin

\section{Introduction}

In recent years multiple studies have been published evaluating the association of obesity classified using the body mass index (BMI) with thyroid function (TF); it has been found a positive correlation between TSH and BMI in most of them. ${ }^{1-4}$ The discrepancies may be in part explained because the body fat percentage $(\mathrm{BF} \%)$ for a given $\mathrm{BMI}$ is highly variable,${ }^{5}$ particularly for individuals in the intermediate BMI ranges; e. gr. for, subjects with BMI of $25 \mathrm{~kg} / \mathrm{m}^{2}$, in men we could find a $\mathrm{BF} \%$ ranging from 13.8 to $35.3 \% \mathrm{BF} \%$, while in women the distribution of $\mathrm{BF} \%$ ranges from 26.4 to $42.8 \%$. $\mathrm{BF} \%$ is currently the gold standard definition of obesity. ${ }^{6}$

In 2007 De Pergola et al., ${ }^{7}$ evaluated the association of TF with waist circumference as an indirect parameter of central fat accumulation. They found that that progressive central fat accumulation is associated with an increase in both FT3 and TSH serum concentrations, independently of insulin sensitivity, metabolic parameters and blood pressure. In another study $\mathrm{T} 3$ and $\mathrm{BF} \%$ have been associated with a significant negative correlation $(\mathrm{r}=-0.37, \mathrm{p}=0.01){ }^{8}$

Another feature often present in overweight and obese subjects is IR. The influence of IR on TF has been addressed in many studies., ${ }^{3,7,9-11}$ In 2010, ${ }^{12}$ Ambrosi and colleagues, published a positive correlation between TSH and Homeostasis Model Assessment of insulin resistance (HOMA-IR) ( $\mathrm{r}=0.14, \mathrm{p}<0.001$ in 581 obese patients), and a negative correlation between FT4 and HOMA-IR $(r=-0.29, p<0.001)$. They suggested that further studies are needed to assess the relation of $\mathrm{TF}$ and body fat distribution.

The aim of this study is to evaluate the association between $\mathrm{TF}$ with $\mathrm{BF} \%$, clinical and biochemical variables including insulin resistance (IR). 


\section{Materials and methods}

\section{Study subjects}

A total of 203 Mexican mestizos were consecutively recruited for this cross-sectional study to determine the association of TF with $\mathrm{BF} \%$ and many other clinical and biochemical variables including IR. The population consisted of subjects from the Internal Medicine and Endocrinology outpatient clinics, of the Instituto Nacional de Ciencias Medicas y Nutricion "Salvador Zubiran" (INCMNSZ) that received attention from March 2007 to July 2010, whom had an indication to receive an OGTT in order to diagnose type 2 diabetes mellitus. We included individuals of both genders from 18 to 65 years-old with a $\mathrm{BMI} \geq 18.0 \mathrm{~kg} / \mathrm{m}^{2}$, and normal $\mathrm{TF}$, determined as a TSH between 0.34 and $5.0 \mathrm{mIU} / \mathrm{mL}$. All patients had a complete measurement of thyroid function: thyroid uptake (TU), thyroid hormone binding ratio $(\mathrm{THBR}=\mathrm{TU} / 0.4)$, total triiodothyronine (T3), total thyroxine (T4), free thyroxine index (FTI=THBR x T4), thyroid stimulating hormone (TSH), thyroglobulin (TG), and an oral glucose tolerance test (OGTT) of 3 hours of duration, using 75 grams of dextrose, with insulin measurement each 30 minutes.

We excluded subjects with known thyroid disease, patients taking thyroid hormones, and medications that could alter the biochemical parameters evaluated such as steroids. We also excluded subjects with other chronic diseases (such as HIV infection or viral hepatitis, lupus or rheumatoid arthritis, seizures, major depression with treatment, hospitalization in last 6 months, active cancer or under treatment), patients with pacemakers, and pregnant women.

The population was divided in men and women for analysis and in obese vs control subjects; obesity was defined in terms of BF\% as published in a position statement by the American Association of Clinical Endocrinologists/American College of Endocrinology. ${ }^{6}$ In men, obesity is defined as a BF\% above $25 \%$, and in women above $35 \%$. Control subjects were not obese with the BF\% definition. Then we compared the clinical and biochemical variables, including TF and IR.

IR was estimated using HOMA-IR ${ }^{13}$ with the formula: glucose $(\mathrm{mg} / \mathrm{dl}) \mathrm{x}$ insulin $(\mu \mathrm{U} / \mathrm{ml}) / 405$; insulin sensitivity was estimated with the ISI Matsuda ${ }^{14}$ with the formula: $10000 / \sqrt{ }($ Glucose 0 ' $x$ Insulin 0') $\mathrm{x}$ (mean glucose $\mathrm{x}$ mean insulin).

\section{Biochemical and anthropometric measurements}

The central laboratory of the INCMNSZ performed all biochemical laboratory measurements. The measurements were performed with commercially available standardized methods. T3, T4, TU and TG were measured by the Access Total assay, a paramagnetic particle, chemiluminescent immunoassay with a Beckman Coulter system, TSH was measured using a third generation assay, a paramagnetic particle, chemiluminescent two-site immunoenzymatic assay with a Beckman Coulter system.

Glucose, total cholesterol, HDL-cholesterol, and triglycerides were measured using the Synchron CX analyzer (Beckman Systems, Fullerton CA). The coefficients of variation for cholesterol and HDL-c were 3.3 and $2.5 \%$, respectively. Plasma insulin concentrations were estimated using a radioimmunoassay method (MEIA, Abbott Laboratories). Anthropometric measurements were done after participants removed their shoes and upper garments. Body weight was measured with a mechanical beam scale (Health o meter Inc,
Bridgeview IL) with daily calibration. Body fat was measured with electrical bioimpedance using a Quantum Desktop-BIA Analyzer by RJL systems. All subjects were instructed to stand in the centre of the scale during weight assessment. Height was obtained using the floor scale's stadiometre. Height was measured to the nearest $0.5 \mathrm{~cm}$. WC was measured to the nearest $0.1 \mathrm{~cm}$ at the level of the greatest frontal extension of the abdomen between the bottom of the rib cage and the top of the iliac crest. BMI was calculated was weight $(\mathrm{kg})$ divided by height $\left(\mathrm{m}^{2}\right)$. Sitting blood pressure was measured after a resting for at least $10 \mathrm{~min}$. This is a sub-analysis of data from an approved study (Validation of a novel adiposity index and its association with insulin resistance), REF: 1650. The human research ethics committee of our institution approved this study. All procedures were done in accordance with the Declaration of Helsinki. Informed consent was obtained from all subjects.

\section{Statistical analysis}

Normally distributed data, determined using KolmogorovSmirnov test are expressed as mean and standard deviation $( \pm \mathrm{SD})$, whereas variables with a skewed distribution were reported as median (interquartil range). The Pearson $\chi^{2}$, Student unpaired $t$-test, or MannWhitney $U$ test was used as appropriate for comparison between men and women, and obese vs control comparisons.

Correlation coefficients between TSH and T3 and the clinical and biochemical variables were evaluated with Pearson or Spearman's test, and partial correlation analysis were made, adjusted for weight (TSH) and weight and age (T3).

A multiple linear regression analysis was done in order to evaluate the variables associated independently with the TSH concentration and in the second multiple regression analysis with variables associated with $\mathrm{T} 3$ concentrations.

All reported $\mathrm{p}$ values are based on two-sided tests considering $\leq 0.05$ as significant. All analyses were performed with SPSS 17.0 (Chicago, IL). The SPSS database is available as a supplementary file.

The SPSS database is available as a supplementary file.

\section{Results}

The basal characteristics of the studied population are shown in Table 1. A total 203 patients (35.9\% male) were included. The mean age of the studied subjects was $38.9 \pm 13.5$ years, with a BMI of $32.7 \pm 8.7 \mathrm{~kg} / \mathrm{m}^{2}$ (range: $18.4-55.8$ ) and a mean $\mathrm{BF} \%$ of $38.4 \pm 9.5 \%$.

Between men and women, there were no differences in T3, T4, free thyroxin index (FTI), and TSH. The characteristics of subjects stratified by sex and $\mathrm{BF} \%$ are shown in Table 2. In men, obese patients had more T3 ( $1.81 \pm 0.53$ vs $1.57 \pm 0.25 \mathrm{nmol} / \mathrm{L}, \mathrm{p}=0.04)$ compared with normal subjects, we did not find a statistically significant differences in TU, THBR, T4, FTI and TG. In women, there were no differences in T3 concentrations $(1.86 \pm 0.49$ vs $1.73 \pm 0.38 \mathrm{nmol} / \mathrm{L}, \mathrm{p}=0.16)$ among obese vs control females, obese women had more TSH $(2.12 \pm 0.9$ vs $1.65 \pm 0.8 \mathrm{mIU} / \mathrm{mL}, \mathrm{p}=0.04$ ); we did not identify a statistically significant difference in TU, THBR, T4, FTI and TG. In both men and women we found a statistically significant difference in weight, BMI, WC, fasting insulin, HOMA-IR, and ISI Matsuda $(\mathrm{p}<0.01)$ between obese and control subjects.

The correlations and partial correlation of TSH and T3, with clinical and biochemical parameters are shown in Table 3. 
Initially we identified a significant correlation between TSH and weight $(\mathrm{r}=0.21, \mathrm{p}=0.002)$, BMI $(\mathrm{r}=0.21, \mathrm{p}=0.001)$, triglyceride concentration $(\mathrm{r}=0.14, \mathrm{p}=0.05)$, and $\mathrm{BF} \%(\mathrm{r}=0.22, \mathrm{p}=0.001)$; after weight adjustment, we identified a significant correlation between $\mathrm{TSH}$ and $\mathrm{BF} \%(\mathrm{r}=0.17, \mathrm{p}=0.02)$, and TSH and $\mathrm{WC}(\mathrm{r}=0.15, \mathrm{P}=0.04)$. We did not find a significant correlation between TSH and HOMA-IR.

Initially $\mathrm{T} 3$ correlated with age $(\mathrm{r}=0.15, \mathrm{p}=0.02)$, weight $(\mathrm{r}=0.16$, $\mathrm{p}=0.02)$, BMI $(\mathrm{r}=0.16, \mathrm{p}=0.02)$, WC $(\mathrm{r}=0.18, \mathrm{p}=0.01)$, triglyceride concentration $(\mathrm{r}=0.16, \mathrm{p}=0.02)$, HDL-C $(\mathrm{r}=-0.22, \mathrm{p}=0.003)$, fasting glucose $(r=0.22, p=0.002)$, fasting insulin $(r=0.31, p<0.001)$, HOMA-
IR $(\mathrm{r}=0.31, \mathrm{p}<0.001)$, and ISI Matsuda $(\mathrm{r}=0.28, \mathrm{p}<0.001)$. And after age and weight adjustment, we identified a significant correlation between T3 and HOMA-IR $(\mathrm{r}=0.38, \mathrm{p}<0.001)$, we also found significant correlations between T3 and HDL-C $(r=0-0.16, p=0.03)$, fasting glucose $(r=0.32, p<0.001)$, fasting insulin $(r=0.33, p<0.001)$, and ISI Matsuda $(r=-025, p<0.001)$. The multiple regression analysis confirmed the previous findings, the variables that were independently associated with TSH concentration were $\mathrm{BF} \%(\beta=0.35, \mathrm{p}<0.001)$ and male gender $(\beta=0.21, \mathrm{p}=0.007)$. The variable independently associated with the T3 concentration was the HOMA-IR $(\beta=6.04$, $\mathrm{p}<0.001$ ) Table 4 .

Table I Anthropometric, clinical and laboratory assessments of the evaluated subjects $(n=203)$

\begin{tabular}{|c|c|c|c|}
\hline Variable & Men $(n=73)$ & Women $(n=130)$ & p value \\
\hline Age (Years) & $39.5 \pm 15.4$ & $38.6 \pm 12.3$ & 0.63 \\
\hline Weight (kg) & $91.9 \pm 20.3$ & $83.1 \pm 21.0$ & 0.004 \\
\hline BMI $\left(\mathrm{kg} / \mathrm{m}^{2}\right)$ & $31.3 \pm 6.9(18.4-51.5)$ & $33.4 \pm 9.5(19.1-55.8)$ & 0.10 \\
\hline$W C(\mathrm{~cm})$ & $105.8 \pm 16.2$ & $103.9 \pm 19.2$ & 0.47 \\
\hline SBP $(\mathrm{mmHg})$ & $122.1 \pm 13.5$ & $114.8 \pm 14.9$ & 0.001 \\
\hline $\mathrm{DBP}(\mathrm{mmHg})$ & $80.2 \pm 11.3$ & $76.3 \pm 10.1$ & 0.01 \\
\hline Total Cholesterol (mg/dL) & $191.3 \pm 34.6$ & $196.2 \pm 35.3$ & 0.36 \\
\hline Triglyceride $(\mathrm{mg} / \mathrm{dL})$ & $218.2(|2| .2-259.0)$ & $147.0(95.0-198.0)$ & 0.13 \\
\hline HDL-C (mg/dL) & $33.3 \pm 8.8$ & $41.3 \pm 10.7$ & $<0.001$ \\
\hline Body Fat (\%) & $31.2 \pm 7.8$ & $42.3 \pm 7.8$ & $<0.001$ \\
\hline Fasting Glucose (mg/dL) & $91.1 \pm 14.1$ & $91.1 \pm 12.0$ & 0.99 \\
\hline Fasting Insulin $(\mu \mathrm{lU} / \mathrm{mL})$ & $9.3(6.3-15.3)$ & $10.4(6.1-14.5)$ & 0.66 \\
\hline HOMA-IR & $2.2(1.3-3.5)$ & $2.3(1.2-3.4)$ & 0.72 \\
\hline ISI-Matsuda & $3.7(2.4-6.5)$ & $3.8(2.3-6.3)$ & 0.93 \\
\hline TU & $0.4 I \pm 0.03$ & $0.40 \pm 0.03$ & 0.03 \\
\hline THBR & $1.03 \pm 0.07$ & $1.0 \pm 0.08$ & 0.03 \\
\hline T3 Total (nmol/L) & $1.7 \pm 0.5$ & $1.7 \pm 0.4$ & 0.81 \\
\hline T4 Total (nmol/L) & $106.7 \pm 25.4$ & $106.5 \pm 22.4$ & 0.95 \\
\hline FTI & $110.9 \pm 29.9$ & $107.2 \pm 25.4$ & 0.36 \\
\hline $\mathrm{TSH}(\mathrm{mlU} / \mathrm{mL})$ & $2.1 \pm 1.0$ & $2.0 \pm 0.9$ & 0.75 \\
\hline TG (ng/mL) & $7.2(3.7-10.7)$ & $6.9(4.0-13.1)$ & 0.73 \\
\hline
\end{tabular}

Data are expressed as mean $\pm \mathrm{SD}$, median (interquartil range), or frequency $(\%)$.

$\mathrm{P}$ values obtained of individual comparison using independent-samples T-Test or Mann-Whitney $\mathrm{U}$ test.

Abbreviations: BMI, body mass index; WC, waist circumference; SBP, systolic blood pressure; DBP, diastolic blood pressure; HDL-C, high density lipoprotein cholesterol; HOMA-IR, homeostasis model assessment insulin resistance; ISI, insulin sensitivity index; TU, thyroid uptake; THBR, thyroid hormone binding ratio: FTI, free thyroxine index; TSH, thyroid stimulating hormone; TG, thyroglobulin. 
Table 2 Patients characteristics stratified by body fat percentage. Obesity defined as (men $>25 \%$, women $>35 \%$ )

\begin{tabular}{|c|c|c|c|c|c|c|}
\hline \multirow{2}{*}{ Variables } & \multicolumn{3}{|l|}{ Men } & \multicolumn{3}{|l|}{ Women } \\
\hline & Control Group $(n=\mid 4)$ & Obese Group $(n=59)$ & p value & $\begin{array}{l}\text { Control } \\
\text { Group }(n=2 I)\end{array}$ & $\begin{array}{l}\text { Obese Group } \\
(n=109)\end{array}$ & $p$ value \\
\hline Age (Years) & $42.2 \pm 17.2$ & $38.9 \pm 15.0$ & 0.47 & $32.6 \pm 13.6$ & $39.7 \pm 11.7$ & 0.01 \\
\hline Weight (kg) & $70.8 \pm 8.4$ & $97.0 \pm 19.1$ & $<0.001$ & $63.2 \pm 11.5$ & $87.0 \pm 20.2$ & $<0.001$ \\
\hline $\mathrm{BMI}\left(\mathrm{kg} / \mathrm{m}^{2}\right)$ & $24.0 \pm 3.3$ & $33.0 \pm 6.3$ & $<0.001$ & $24.0 \pm 3.6$ & $35.2 \pm 9.3$ & $<0.001$ \\
\hline WC (cm) & $88.0 \pm 9.3$ & $103.5 \pm 16.6$ & $<0.001$ & $82.8 \pm 11.7$ & $107.9 \pm 17.7$ & $<0.001$ \\
\hline $\mathrm{SBP}(\mathrm{mmHg})$ & $1 \mid 4.7 \pm 13.7$ & $123.8 \pm 13.0$ & 0.02 & $108.9 \pm 10.8$ & $116.0 \pm 15.4$ & 0.04 \\
\hline $\mathrm{DBP}(\mathrm{mmHg})$ & $73.9 \pm 17.9$ & $81.7 \pm 8.6$ & 0.02 & $73.6 \pm 7.2$ & $76.9 \pm 10.5$ & 0.17 \\
\hline $\begin{array}{l}\text { Total Cholesterol } \\
(\mathrm{mg} / \mathrm{dL})\end{array}$ & $175.9 \pm 38.3$ & $194.6 \pm 33.1$ & 0.13 & $176.0 \pm 36.5$ & $199.8 \pm 34.1$ & 0.01 \\
\hline Triglyceride (mg/dL) & | $3 \mid .5(64.2-278.5)$ & | 48.5 (|23.0-259.0) & 0.26 & $80.0(60.0-168.0)$ & I53.5 (I05.2-202.5) & 0.03 \\
\hline HDL-C (mg/dL) & $35.1 \pm 7.6$ & $33.0 \pm 9.1$ & 0.31 & $46.1 \pm 11.7$ & $40.4 \pm 10.3$ & 0.04 \\
\hline Body Fat (\%) & $20.2 \pm 4.0$ & $33.8 \pm 6.1$ & $<0.001$ & $29.9 \pm 3.2$ & $44.7 \pm 5.9$ & $<0.001$ \\
\hline $\begin{array}{l}\text { Fasting Glucose (mg/ } \\
\mathrm{dL} \text { ) }\end{array}$ & $87.2 \pm 9.2$ & $92.0 \pm 15.0$ & 0.35 & $86.5 \pm 10.3$ & $91.9 \pm 12.1$ & 0.056 \\
\hline $\begin{array}{l}\text { Fasting Insulin }(\mu \mathrm{lU} / \\
\mathrm{mL})\end{array}$ & $5.8(4.4-8.4)$ & $11.9(7.2-18.3)$ & 0.002 & $4.8(3.1-8.9)$ & II.2(7.1-I5.7) & $<0.001$ \\
\hline HOMA-IR & I.I (0.8-2.0) & $2.4(1.6-4.0)$ & 0.003 & $0.9(0.6-2.0)$ & $2.6(1.4-3.6)$ & $<0.001$ \\
\hline ISI-Matsuda & $7.5(4.3-12.1)$ & $3.2(2.1-5.5)$ & 0.001 & 7.1 (4.7-II.9) & $3.5(2.2-5.5)$ & $<0.001$ \\
\hline TU & $0.4 I \pm 0.03$ & $0.4 I \pm 0.03$ & 0.93 & $0.40 \pm 0.03$ & $0.40 \pm 0.03$ & 0.82 \\
\hline THBR & $1.03 \pm 0.07$ & $1.03 \pm 0.08$ & 0.93 & $1.01 \pm 0.09$ & $1.00 \pm 0.08$ & 0.82 \\
\hline T3 Total (nmol/L) & $1.57 \pm 0.25$ & $\mathrm{I} .8 \mathrm{I} \pm 0.53$ & 0.04 & $1.86 \pm 0.49$ & $1.73 \pm 0.38$ & 0.16 \\
\hline T4 Total (nmol/L) & $105.6 \pm 29.3$ & $107.0 \pm 24.7$ & 0.72 & $111.0 \pm 16.8$ & $105.6 \pm 23.3$ & 0.31 \\
\hline FTI & $109.23 \pm 35.37$ & $111.3 \pm 28.9$ & 0.82 & $112.1 \pm 19.7$ & $106.2 \pm 26.4$ & 0.33 \\
\hline TSH (mIU/mL) & $1.6 \pm 0.9$ & 2. $I \pm 1.0$ & 0.1 & $1.65 \pm 0.8$ & $2.12 \pm 0.9$ & 0.04 \\
\hline TG (ng/mL) & $6.9(3.4-10.5)$ & $7.5(3.7-10.8)$ & 0.93 & $5.9(3.2-9.7)$ & $7.0(4.3-13.7)$ & 0.11 \\
\hline
\end{tabular}

Data are expressed as mean \pm SD, median (interquartil range), or frequency (\%).

$P$ values obtained of individual comparison using independent-samples T-Test or Mann-Whitney $U$ test.

Abbreviations: BMI, body mass index;WC, waist circumference; SBP, systolic blood pressure; DBP, diastolic blood pressure; HDL-C, high density lipoprotein cholesterol; HOMA-IR, homeostasis model assessment insulin resistance; ISI, insulin sensitivity index; TU, thyroid uptake; THBR, thyroid hormone binding ratio; FTI, free thyroxine index; TSH, thyroid stimulating hormone; TG, thyroglobulin. 
Table 3 Partial correlations of TSH and total T3 with clinical and biochemical parameters evaluated in the sample studied $(\mathrm{n}=203)$

\begin{tabular}{|c|c|c|c|c|c|c|c|c|}
\hline \multirow{2}{*}{ Variable } & \multicolumn{2}{|l|}{ TSH } & \multicolumn{2}{|l|}{ T3 } & \multicolumn{2}{|c|}{ TSH (Weight adjusted) } & \multicolumn{2}{|c|}{ T3 (Age, and weight adjusted) } \\
\hline & $\mathbf{r}$ & $p$ value & $\mathbf{r}$ & p value & $\mathbf{r}$ & $\mathrm{p}$ value & $\mathbf{r}$ & p value \\
\hline Age (Years) & 0.05 & 0.41 & 0.15 & 0.02 & 0.09 & 0.23 & - & - \\
\hline Sex & 0.02 & 0.72 & 0.01 & 0.79 & -0.04 & 0.55 & 0.03 & 0.61 \\
\hline Weight (kg) & 0.21 & 0.002 & 0.16 & 0.02 & - & - & - & - \\
\hline BMI $\left(\mathrm{kg} / \mathrm{m}^{2}\right)$ & 0.22 & 0.001 & 0.16 & 0.02 & 0.09 & 0.22 & 0.05 & 0.45 \\
\hline$W C(\mathrm{~cm})$ & 0.24 & $<0.001$ & 0.18 & 0.01 & 0.15 & 0.04 & 0.14 & 0.06 \\
\hline $\mathrm{SBP}(\mathrm{mmHg})$ & 0.10 & 0.15 & 0.05 & 0.44 & 0.04 & 0.55 & 0.07 & 0.34 \\
\hline $\mathrm{DBP}(\mathrm{mmHg})$ & 0.11 & 0.09 & 0.05 & 0.44 & 0.06 & 0.38 & 0.01 & 0.88 \\
\hline Total Cholesterol (mg/dL) & 0.08 & 0.26 & -0.05 & 0.45 & 0.07 & 0.32 & -0.007 & 0.92 \\
\hline Triglyceride (mg/dL) & 0.14 & 0.05 & 0.16 & 0.02 & -0.009 & 0.90 & 0.06 & 0.37 \\
\hline HDL-C (mg/dL) & -0.02 & 0.97 & -0.22 & 0.003 & 0.03 & 0.66 & -0.16 & 0.03 \\
\hline Body Fat (\%) & 0.22 & 0.001 & 0.11 & 0.10 & 0.17 & 0.02 & -0.01 & 0.90 \\
\hline Fasting Glucose (mg/dL) & -0.03 & 0.66 & 0.22 & 0.002 & -0.03 & 0.64 & 0.32 & $<0.001$ \\
\hline Fasting Insulin $(\mu \mathrm{lU} / \mathrm{mL})$ & 0.12 & 0.07 & 0.31 & $<0.001$ & -0.01 & 0.89 & 0.33 & $<0.001$ \\
\hline HOMA-IR & 0.11 & 0.09 & 0.31 & $<0.001$ & -0.02 & 0.70 & 0.38 & $<0.001$ \\
\hline ISI Matsuda & 0.11 & 0.10 & -0.28 & $<0.001$ & -0.06 & 0.41 & -0.25 & 0.001 \\
\hline
\end{tabular}

P values obtained of individual comparison using Spearman or Pearson bivariate correlation tests.

Abbreviations, BMI, body mass index; WC, waist circumference; SBP, systolic blood pressure; DBP, diastolic blood pressure; HDL-C, high density lipoprotein cholesterol; HOMA-IR, homeostasis model assessment insulin resistance; ISI, insulin sensitivity index; TSH, thyroid stimulating hormone; T3, Total T3.

Table 4 Multiple linear regression analysis of variables associated with TSH and T3 concentrations.

Dependent Variable TSH concentration,

\begin{tabular}{llll}
\hline Independent variable & $\boldsymbol{\beta}$ coefficient & $\mathbf{t}$ & $\mathbf{p}$ value \\
$\mathrm{BF} \%$ & 0.35 & 4.34 & $<0.00 \mathrm{I}$ \\
Age & 0.005 & 0.98 & 0.32 \\
Gender (male) & 0.21 & 2.7 & 0.007 \\
\hline
\end{tabular}

Parameters of the model, $R 2=0.091, F=6.57, p<0.001$.

Abbreviation: BF\%, Body Fat Percentage.

Dependent variable T3

\begin{tabular}{llll}
\hline Independent Variable & $\boldsymbol{\beta}$ Coefficient & $\mathbf{t}$ & $\boldsymbol{p}$ value \\
\hline Age & -0.11 & -1.79 & 0.075 \\
Gender & -0.011 & -0.17 & 0.864 \\
HOMA-IR & 0.399 & 6.04 & $<0.001$ \\
\hline
\end{tabular}

Parameters of the model, $R^{2}=0.18, F=16.16, p<0.001$.

Abbreviation: HOMA-IR, Homeostasis Model Assessment of insulin resistance. 


\section{Discussion}

The aim of this study was to evaluate the association between $\mathrm{TF}$ and $\mathrm{BF} \%$. We also evaluated the association of TF with clinical, anthropometric and biochemical variables including insulin resistance (IR). We identified that in men, obese patients have more T3, and a tendency towards more TSH; in women, there were no differences in $\mathrm{T} 3$ concentrations among obese vs control females, and obese women have more TSH compared to normal women.

After weight adjustment, we identified a significant correlation between $\mathrm{TSH}$ and $\mathrm{BF} \%$, and TSH with WC.

After age and weight adjustment, we identified a significant correlation between T3 and HOMA-IR, HDL-C, fasting glucose, fasting insulin, and ISI Matsuda. In the multiple regression analysis $\mathrm{BF} \%$ and male gender, were independently associated with TSH concentration, and HOMA-IR was independently associated with T3 concentration.

Regarding $\mathrm{BF} \%$ and $\mathrm{TF}$, one $\mathrm{study}^{8}$ reported a significant correlation between $\mathrm{T} 3$ and $\mathrm{BF} \%(\mathrm{r}=-0.37, \mathrm{p}=0.012)$, and nonsignificant correlations between $\mathrm{BF} \%$ and $\mathrm{T} 4, \mathrm{TSH}$, and Free T3, another study ${ }^{15}$ showed a significant correlation between $\mathrm{BF} \%$ and TSH $(r=0.13, p=0.03$, Free T3 $(r=-0.15, p=0.02)$ and Free T4 $(r=-$ $0.20, \mathrm{p}=0.001)$; however in both studies, the correlation analysis were not adjusted for body weight. Another study ${ }^{16}$ that assessed the relationship of TSH with body fat, showed a positive correlation of TSH and body fat weight $(r=0.268, p=0.038)$.

Our study results, after weight adjustment, showed only a significant correlation between $\mathrm{BF} \%$ and TSH $(r=0.17, p=0.02)$ and no association between $\mathrm{T} 3$ and $\mathrm{BF} \%$. We believe that associations of TSH with obesity should be done using $\mathrm{BF} \%$ as obesity definition, instead of BMI, because the $\mathrm{BF} \%$ for a given $\mathrm{BMI}$ is highly variable. ${ }^{5}$ One study in a Hispanic population ${ }^{17}$ also showed a positive correlation between TSH (adjusted for age and sex) with waist circumference $(\beta=0.10, p<0.001)$.

One possible explanation of the positive correlation between TSH and $\mathrm{BF} \%$ is that obese patients have increased concentrations of leptin and pro-opiomelanocortin which directly stimulates thyrotropin releasing hormone (TRH) neurons in the paraventricular nucleus, leading to an increment in TSH. ${ }^{18,19}$ We need prospective cohort studies to know if obesity per se may influence thyroid function or whether small differences in thyroid function, even in a range of normally, might affect body weight.

The influence of IR on TF has been addressed in many studies., ,, $9-12^{-12}$ In our study, after age and weight adjustment, we identified a significant correlation between T3 and HOMA-IR, HDL-C, fasting glucose, fasting insulin, and ISI Matsuda. We did not find a significant correlation between TSH and HOMA-IR, after weight adjustment because body weight has also a positive correlation with HOMAIR $(\mathrm{r}=0.54, \mathrm{p}<0.001, \mathrm{n}=623$; unpublished data, from our cohort of patients). The positive correlation of T3 with HOMA-IR and insulin that we found could be explained by the fact that plasma-free T3 concentrations may play a role in the regulation of insulin secretion; ${ }^{20}$ $\mathrm{T} 3$ enhances insulin release in isolated rat pancreatic islets at certain glucose concentrations. ${ }^{21}$

Some studies have reported that metformin decreases $\mathrm{TSH},{ }^{22,23}$ since IR also affects TF, further studies should be done to assess the effect of metformin in TSH after IR adjustment.

This study has some limitations, firstly this was a cross sectional study, so TF over time cannot be assessed, secondly, we used surrogate indexes to measure insulin resistance, we included only Mexican mestizos, and finally we did not measure urine iodine as a marker of iodine deficiency in our population.

In conclusion we found that TSH is associated with $\mathrm{BF} \%$ independently of weight and T3 is associated with HOMA-IR, independently of age and weight.

\section{Acknowledgements}

MAGS express his deep gratitude to Luz del Carmen Abascal Olascoaga.

\section{Conflicts of interest}

The author declares there is no conflict of interest.

\section{References}

1. Knudsen N, Laurberg P, Rasmussen LB, et al. Small differences in thyroid function may be important for body mass index and the occurrence of obesity in the population. $J$ Clin Endocrinol Metab. 2005;90(7):4019-4024.

2. Nyrnes A, Jorde R, Sundsfjord J. Serum TSH is positively associated with BMI. Int J Obes (Lond). 2006;30(1):100-105.

3. Michalaki MA, Vagenakis AG, Leonardou AS, et al. Thyroid function in humans with morbid obesity. Thyroid. 2006;16(1):73-78.

4. Manji N, Boelaert K, Sheppard MC, et al. Lack of association between serum TSH or free T4 and body mass index in euthyroid subjects. Clin Endocrinol (Oxf). 2006;64(2):125-128.

5. Romero-Corral A, Somers VK, Sierra-Johnson J, et al. Accuracy of body mass index in diagnosing obesity in the adult general population. Int J Obes (Lond). 2008;32(6):959-966.

6. Dickey R, Bartuska DG, Bray GW, et al. AACE/ACE position statement on the prevention, diagnosis and treatment of obesity. Endocr Pract. 1998;4(5):297-350.

7. De Pergola G, Ciampolillo A, Paolotti S, et al. Free triiodothyronine and thyroid stimulating hormone are directly associated with waist circumference, independently of insulin resistance, metabolic parameters and blood pressure in overweight and obese women. Clin Endocrinol (Oxf). 2007;67(2):265-269.

8. Kumar HK, Yadav RK, Prajapati J, et al. Association between thyroid hormones, insulin resistance, and metabolic syndrome. Saudi Med J. 2009;30(7):907-911.

9. Iacobellis G, Ribaudo MC, Zappaterreno A, et al. Relationship of thyroid function with body mass index, leptin, insulin sensitivity and adiponectin in euthyroid obese women. Clin Endocrinol (Oxf). 2005;62(4):487-491.

10. Roos A, Bakker SJ, Links TP, et al. Thyroid function is associated with components of the metabolic syndrome in euthyroid subjects. $J$ Clin Endocrinol Metab. 2007;92(2):491-496.

11. Bastemir M, Akin F, Alkis E, et al. Obesity is associated with increased serum TSH level, independent of thyroid function. Swiss Med Wkly. 2007;137(29-30):431-434.

12. Ambrosi B, Masserini B, Iorio L, et al. Relationship of thyroid function with body mass index and insulin-resistance in euthyroid obese subjects. J Endocrinol Invest. 2010;33(9):640-643.

13. Matthews DR, Hosker JP, Rudenski AS, et al. Homeostasis model assessment: insulin resistance and -cell function from fasting plasma glucose and insulin concentrations in man. Diabetologia. 1985;28(7):412419. 
14. Matsuda M, DeFronzo RA. Insulin sensitivity indices obtained from oral glucose tolerance testing: comparison with the euglycemic insulin clamp. Diabetes Care. 1999;22(9):1462-1470.

15. Marzullo P, Minocci A, Tagliaferri MA, et al. Investigations of thyroid hormones and antibodies in obesity: leptin levels are associated with thyroid autoimmunity independent of bioanthropometric, hormonal, and weight-related determinants. J Clin Endocrinol Metab. 2010;95(8):3965-3972.

16. Sari R, Balci MK, Altunbas $\mathrm{H}$, et al. The effect of body weight and weight loss on thyroid volume and function in obese women. Clin Endocrinol (Oxf). 2003;59(2):258-262.

17. Garduño-Garcia Jde J, Alvirde-Garcia U, López-Carrasco G, et al. TSH and free thyroxine concentrations are associated with differing metabolic markers in euthyroid subjects. Eur $J$ Endocrinol. 2010;163(2):273-278.

18. Mihály E, Fekete C, Tatro JB, et al. Hypophysiotropic thyrotropinreleasing hormone-synthesizing neurons in the human hypothalamus are innervated by neuropeptide Y, agouti-related protein, and alpha-melanocyte-stimulating hormone. J Clin Endocrinol Metab. 2000;85(7):2596-2603.
19. Pinkney JH, Goodrick SJ, Katz J, et al. Leptin and the pituitary-thyroid axis: a comparative study in lean, obese, hypothyroid and hyperthyroid subjects. Clin Endocrinol (Oxf). 1998;49(5):583-588.

20. Ortega E, Koska J, Pannacciulli N, et al. Free triiodothyronine plasma concentrations are positively associated with insulin secretion in euthyroid individuals. Eur J Endocrinol. 2008;158(2):217-221.

21. Cortizo AM, Chazenbalk GD, de Gagliardino EE, et al. Thyroid hormone binding and deiodination by pancreatic islets: relationship with the in vitro effect upon insulin secretion. Acta Endocrinol (Copenh). 1987;116(1):66-72.

22. Cappelli C, Rotondi M, Pirola I, et al. TSH-lowering effect of metformin in type 2 diabetic patients: differences between euthyroid, untreated hypothyroid, and euthyroid on L-T4 therapy patients. Diabetes Care. 2009;32(9):1589-1590.

23. Morteza Taghavi S, Rokni H, et al. Metformin decreases thyrotropin in overweight women with polycystic ovarian syndrome and hypothyroidism. Diab Vasc Dis Res. 2011;8(1): 47-48. 